\title{
Numerical Simulation of Coupled Fluid-Solid Systems by Fictitious Boundary and Grid Deformation Methods
}

\author{
Decheng $\mathrm{Wan}^{1}$ and Stefan Turek ${ }^{2}$ \\ Institute of Applied Mathematics LS III, University of Dortmund, \\ Vogelpothsweg 87, 44227 Dortmund, Germany \\ ${ }^{1}$ wan.decheng@math. uni-dortmund.de ${ }^{2}$ stefan.turek@math.uni-dortmund.de
}

Summary. Numerical simulations of coupled fluid-rigid solid problems by multigrid fictitious boundary and grid deformation methods are presented. The flow is computed by a special ALE formulation with a multigrid finite element solver. The solid body is allowed to move freely through the computational mesh which is adaptively aligned by a special mesh deformation method such that the accuracy for dealing with the interaction between the fluid and the solid body is highly improved. Numerical examples are provided to show the efficiency of the presented method.

\section{Introduction}

Efficient numerical solution of the coupled fluid-solid system is still a challenging task in many applications and a topic of current mathematical research. Different differential equations must be satisfied on each side of the interface between fluid and rigid body and the solutions are coupled through relationships or jump conditions that must hold at the interface. The movement of both the interface and the rigid body is unknown in advance and must be determined as part of the solution.

There are two types of methods to meet this challenge. The first approach is a generalized standard Galerkin finite element method [1,2] in which both the fluid and solid equations of motion are incorporated into a single coupled variational equation. The computation is performed on an unstructured body-fitted grid, and an arbitrary Lagrangian-Eulerian (ALE) moving mesh technique is adopted to deal with the motion of the solid. In the case of $2 \mathrm{D}$, the remeshing of body-fitted grid can be done by many available grid generation software tools, but in the more interesting case of a full 3D simulation, the problem of efficient, body-fitted grid generation is not solved in a satisfying manner yet. The second approach is based on the principle of embedded or fictitious domains, in which the fluid flow is computed as if the space occupied by the solid were filled with fluid, and the no-slip boundary condition 
on the solid boundaries is enforced as a constraint. The fictitious domain is discretized only once in the beginning. For example, the distributed Lagrange multiplier (DLM)/fictitious domain method developed by Glowinski, Joseph and coauthors [3], and our multigrid fictitious boundary method (FBM) $[4,5]$ belong to this class. An advantage of the fictitious domain method over the generalized standard Galerkin finite element method is that the fictitious domain method allows a fixed grid to be used, eliminating the need for remeshing. An underlying problem when adopting the fictitious domain method is that the boundary approximation is of low accuracy. One remedy could be to preserve the mesh topology, for instance as generalized tensorproduct or blockstructured meshes, while a local alignment with the physical boundary of the solid is achieved by a grid deformation process, such that the boundary approximation error can be significantly decreased.

Over the past decade, several grid adaptation techniques have been developed, namely the so-called $h$-, $p$ - and $r$-methods. The first two do static remeshing, where the $h$-method does automatic refinement or coarsening of the spatial mesh based on a posteriori error estimates or error indicators and the $p$-method takes higher or lower order approximations locally as needed. In contrast, the $r$-method (also known as moving grid method) relocates grid points in a mesh having a fixed number of nodes in such a way that the nodes remain concentrated in regions of rapid variation of the solution or corresponding interfaces. The $r$-method is a dynamic method which means that it uses time stepping or pseudo-time stepping approaches to construct the desired transformation. Compared to the $h$ - and $p$-methods in which often hanging nodes have to be dealt with, the $r$-method is much easier to incorporate into most CFD codes without the need for changing of system matrix structures and special interpolation procedures since in the $r$-method the data structures for mesh are fixed. The $r$-method has received considerably attention recently due to some new developments which clearly demonstrate its potential for problems such as those having moving interfaces $[6,7]$.

In this paper, we base on our multigrid fictitious boundary method (FBM) $[4,5]$ and the grid deformation method presented in [7] to solve numerically the coupled fluid-solid problems. As we have shown in [5], the use of the multigrid FBM does not require to change the mesh during the simulations when the solid bodies vary their positions. The advantage is that no expensive remeshing has to be performed while a fixed mesh can be used such that in combination with appropriate data structures and fast CFD solvers very high efficiency rates can be reached. However, the accuracy for capturing the surfaces of solid bodies is only of first order which might lead to accuracy problems. For a better approximation of the solid surfaces, we adopt a deformed grid, created from an equidistant cartesian mesh in which the topology is preserved and only the grid spacing is changed such that the grid points are concentrated near the surfaces of the solid bodies. Here, only the solution of additional linear Poisson problems in every time step is required for generating the deformation grid, which means that the additional work 
is significantly less than the main fluid-solid part. The presented method is compared to the pure multigrid FBM using an equidistance mesh through a numerical simulation of a benchmark configuration of $2 \mathrm{D}$ flow around an airfoil in a channel. Its accuracy improvement is shown to be excellent.

\section{Grid Deformation Method}

In this section, we briefly describe the grid deformation method which will be adopted and coupled with our multigrid fictitious boundary method (FBM) (see the next section) to solve numerically the fluid-rigid solid problems. The details of the grid deformation method can be found in [7].

Grid deformation problems can be equated to construct a transformation $\phi, x=\phi(\xi)$ from the computational space (with coordinate $\xi$ ) to the physical space (with coordinate $x$ ). There are two basic types of grid deformation methods, local based and velocity based, generally computing $x$ by minimizing a variational form or computing the mesh velocity $v=x_{t}$ using a Lagrangianlike formulation. The grid deformation method we will employ belongs to the velocity-based method, which is based on Liao's work [6] and Moser's work [8]. This method has several advantages: only linear Poisson problems on fixed meshes are needed to be solved, the monitor function can be obtained directly from an error distribution, mesh tangling can be prevented, and the data structure is always the same as that for the starting mesh (see [7]).

Suppose $g(x)$ (area function) to be the area distribution on the undeformed mesh, while $f(x)$ (monitor function) in contrast describes the absolute mesh size distribution of the target grid, which is independent of the starting grid and chosen according to the need of physical problems. Then, the transformation $\phi$ can be computed via the following four steps:

1. Compute the scale factors $c_{f}$ and $c_{g}$ for the given monitor function $f(x)>$ 0 and the area function $g$ using

$$
c_{f} \int_{\Omega} \frac{1}{f(x)} d x=c_{g} \int_{\Omega} \frac{1}{g(x)} d x=|\Omega|,
$$

where, $\Omega \subset \mathbb{R}^{2}$ is a computational domain, and $f(x) \approx$ local mesh area. Let $\tilde{f}$ and $\tilde{g}$ denote the reciprocals of the scaled functions $f$ and $g$, i.e.,

$$
\tilde{f}=\frac{c_{f}}{f}, \quad \tilde{g}=\frac{c_{g}}{g} .
$$

2. Compute a grid-velocity vector field $v: \Omega \rightarrow \mathbb{R}^{n}$ by satisfying the following linear Poisson equation

$$
-\operatorname{div}(v(x))=\tilde{f}(x)-\tilde{g}(x), \quad x \in \Omega, \quad \text { and } \quad v(x) \cdot \mathfrak{n}=0, \quad x \in \partial \Omega,
$$

where $\mathfrak{n}$ being the outer normal vector of the domain boundary $\partial \Omega$, which may consist of several boundary components. 
3. For each grid point $x$, solve the following ODE system

$$
\frac{\partial \varphi(x, t)}{\partial t}=\eta(\varphi(x, t), t), \quad 0 \leq t \leq 1, \quad \varphi(x, 0)=x,
$$

with

$$
\eta(y, s):=\frac{v(y)}{s \tilde{f}(y)+(1-s) \tilde{g}(y)}, \quad y \in \Omega, \quad s \in[0,1] .
$$

4. Get the deformed grid points via

$$
\phi(x):=\varphi(x, 1) .
$$

\section{Numerical Solution of the Fluid-Solid System}

In this section, we will describe how to solve numerically the coupled fluid-rigid solid problems by using our multigrid FBM and the above grid deformation method.

\subsection{Governing Equations in the Frame of FBM}

For a detailed description of the multigrid fictitious boundary method (FBM), the reader is referred to Refs. $[4,5]$. The governing coupled fluid-solid system in the frame of the FBM can be given by

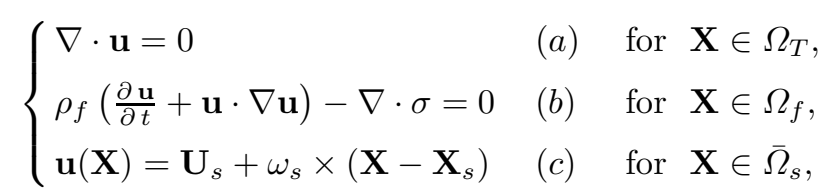

where $\sigma$ is the total stress tensor in the fluid phase defined as

$$
\sigma=-p \mathbf{I}+\mu_{f}\left[\nabla \mathbf{u}+(\nabla \mathbf{u})^{T}\right] .
$$

Here $\mathbf{I}$ is the identity tensor, fluid viscosity $\mu_{f}=\rho_{f} \cdot \nu, \rho_{f}$ is the fluid density, $p$ is the pressure and $\mathbf{u}$ is the fluid velocity. $\Omega_{f}$ is the domain occupied by the fluid, and $\Omega_{s}$ the domain occupied by the rigid bodies, $\Omega_{T}=\Omega_{f} \cup \Omega_{s}$ the entire computational domain.

The equations that govern the motion of the rigid body are the following Newton-Euler equations, i.e., the translational velocities $\mathbf{U}_{s}$ and angular velocities $\omega_{s}$ of the rigid body satisfy

$$
M_{s} \frac{d \mathbf{U}_{s}}{d t}=\left(\Delta M_{s}\right) \mathbf{g}+\mathbf{F}_{s}, \quad \mathbf{I}_{s} \frac{d \omega_{s}}{d t}+\omega_{s} \times\left(\mathbf{I}_{s} \omega_{s}\right)=T_{s},
$$

where $M_{s}$ is the mass of the rigid body; $\mathbf{I}_{s}$ is the moment of the inertia tensor; $\Delta M_{s}$ is the mass difference between the mass $M_{s}$ and the mass of 
the fluid occupying the same volume; $\mathbf{g}$ is the gravity vector; $\mathbf{F}_{s}$ and $T_{s}$ are the resultants of the hydrodynamic forces and the torque about the center of mass acting on the rigid body which are calculated by

$$
\mathbf{F}_{s}=-\int_{\Omega_{T}} \sigma \cdot \nabla \alpha_{s} d \Omega, \quad T_{s}=-\int_{\Omega_{T}}\left(\mathbf{X}-\mathbf{X}_{s}\right) \times\left(\sigma \cdot \nabla \alpha_{s}\right) d \Omega .
$$

Here the function $\alpha_{s}$ is defined by

$$
\alpha_{s}(\mathbf{X})=\left\{\begin{array}{lll}
1 & \text { for } & \mathbf{X} \in \bar{\Omega}_{s}, \\
0 & \text { for } & \mathbf{X} \in \Omega_{T} \backslash \Omega_{s} .
\end{array}\right.
$$

The position $\mathbf{X}_{s}$ of the rigid body and its angle $\theta_{s}$ are obtained by integration of the kinematic equations

$$
\frac{d \mathbf{X}_{s}}{d t}=\mathbf{U}_{s}, \quad \frac{d \theta_{s}}{d t}=\omega_{s}
$$

\subsection{ALE Formulation}

When the grid deformation method described in section 2 is applied to the multigrid FBM, a mesh velocity $\mathbf{W}_{m}$ should be introduced in the convective term of Eq. (7b), i.e.,

$$
\rho_{f}\left[\frac{\partial \mathbf{u}}{\partial t}+\left(\mathbf{u}-\mathbf{W}_{m}\right) \cdot \nabla \mathbf{u}\right]-\nabla \cdot \sigma=0 \quad \text { for } \mathbf{X} \in \Omega_{f} .
$$

In the literature this is referred to as an Arbitrary Lagrangian-Eulerian (ALE) formulation. Note that the mesh velocities $\mathbf{W}_{m}$ do not appear in the continuity equation, as a pressure-Poisson equation is solved to satisfy the continuity equation in an outer loop. Care has to be taken to satisfy the geometric conservation law (GCL), where the mesh velocity $\mathbf{W}_{m}$ must be equal to the movement of the mesh velocity $\Delta \mathrm{x}$ during the time step. Therefore, the mesh velocities $\mathbf{W}_{m}$ should be calculated according to the nodal movement from the previous time step by

$$
\mathbf{W}_{m}=\frac{1}{\Delta t}\left(\mathbf{x}^{n+1}-\mathbf{x}^{n}\right),
$$

where $\Delta t$ is the time step size $t^{n+1}-t^{n}$.

\subsection{Numerical Realization in FEATFLOW}

A special solver has been developed to solve the coupled fluid-rigid solid problems in the ALE formulation by using the multigrid FBM and the grid deformation method. It is essentially based on the discrete projection type solver PP2D from the FEATFLOW package $[9,10]$. 
In this solver, we first semi-discretize in time by a Fractional-step- $\theta$-scheme (see [9]). Then, we obtain a sequence of generalized stationary Navier-Stokes equations with both prescribed boundary values and fictitious boundary conditions for the moving rigid body in the fluid in every time step, which is a nonlinear saddle point problem that has to be discretized in space. For the spatial discretization, we choose the nonconforming $\tilde{Q}_{1} / Q_{0}$ element pair, in which the nodal values are the mean values of the velocity over the element edges and the mean values of the pressure over the elements. The non-linear one-step projection solution process is accelerated with a multigrid technique [9]. In each time step, a new deformation mesh is generated based on the starting mesh and two auxiliary routines are designed to update the system matrices and to calculate the mesh velocity according to the new position of the deformation mesh nodes, respectively. After the fluid part calculation, we can do the solid part calculation, including the calculation of the corresponding hydrodynamic forces and the torque acting on the rigid body as well as the updating of the new positions and velocities of the solid body.

\section{Verification of the Numerical Techniques}

The new solver is validated in this section by a benchmark configuration of 2D flow around an airfoil in a channel (see [3] for the details) to assess the accuracy and efficiency of the proposed combination based on the multigrid FBM and the grid deformation method compared to the results without using the grid deformation method.

We consider a NACA0012 airfoil that has a fixed center of mass and is induced to rotate freely around its center of mass due to hydrodynamical forces under the action of an incoming incompressible viscous flow in a channel. The channel is of width 20 and height 4 . All values are in non-dimensional form. The density of the fluid is $\rho_{f}=1$ and the density of the airfoil is $\rho_{p}=1.1$. The viscosity of the fluid is $\nu_{f}=10^{-2}$. The initial condition for the fluid velocity is $\mathbf{u}=0$ and the boundary conditions are given as $\mathbf{u}=0$ when $y=0$ or 4 and $\mathbf{u}=1$ when $x=0$ or 20 for $t \geq 0$. Initial angular velocity and angle of incidence of the airfoil are zero. The airfoil length is 1.0089304 and the fixed center of mass of the airfoil is at $(0.420516,2)$. Hence the Reynolds number is about 101 with respect to the length of the airfoil and the inflow speed. The detailed shape of the NACA0012 is described as follows (for $0 \leq X \leq 1.0089304$ ):

$Y=0.6 \cdot\{0.2969 \cdot \sqrt{X}+X \cdot[-0.126+X \cdot[-0.3516+X \cdot(0.2843-0.1015 \cdot X)]]\}$

The simulation is implemented on both fixed equidistance meshes and moving deformation meshes, each of them for two different levels, i.e., Level $=$ 7 with 41409 nodes and 40960 elements, as well as Level $=8$ with 164737 nodes and 163840 elements. The deformation mesh is generated in each time step in order to always keep grid alignment near the surface of the induced rotating 


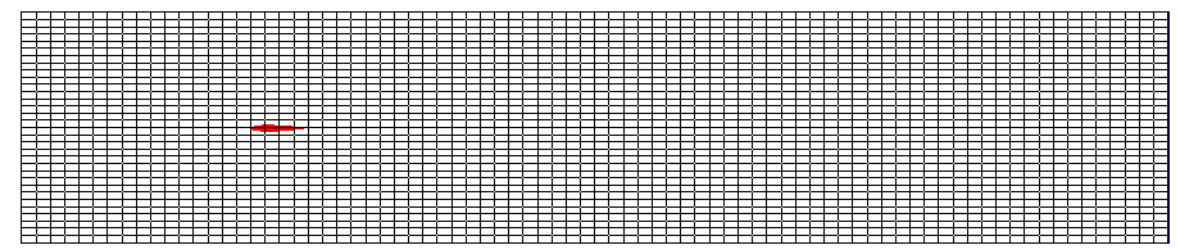

(a) Starting mesh

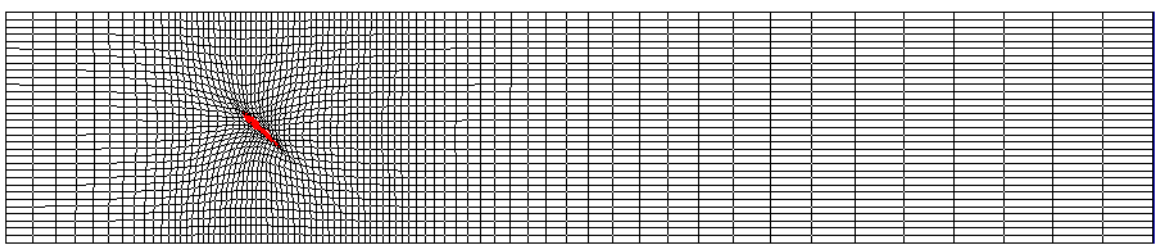

(b) Deformation mesh $(t=14.7)$

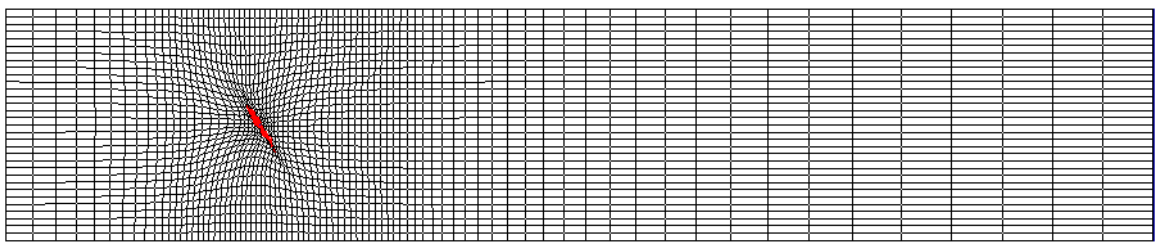

(c) Deformation mesh $(t=16.0)$

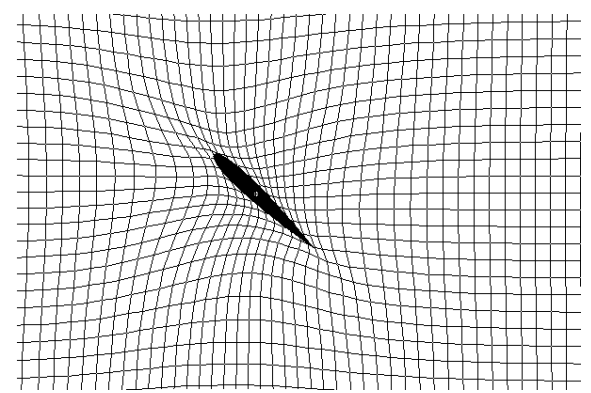

(d) Zoom mesh $(t=14.7)$

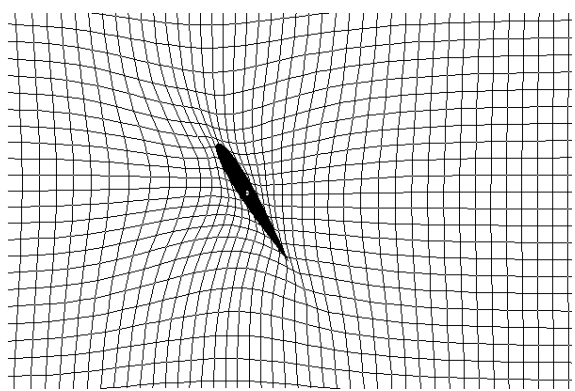

(e) Zoom mesh $(t=16.0)$

Fig. 1. Starting mesh and deformation meshes employed during the simulation of the induced rotation of a NACA0012 airfoil in a channel

airfoil. Fig. 1 (a) shows the starting equidistance mesh used to generate deformation meshes. In Fig. 1 (b) and (c), two deformation meshes at $t=14.7$ and $t=16.0$ are presented, their local zoom meshes are illustrated in Fig. 1 (d) and (e). Fig. 2 plots the time history of the angle of incidence $\theta$ and the angular velocity $\omega$ of the airfoil calculated by using equidistance meshes and deformation meshes, each of them is performed by two levels LEVEL $=7$ 


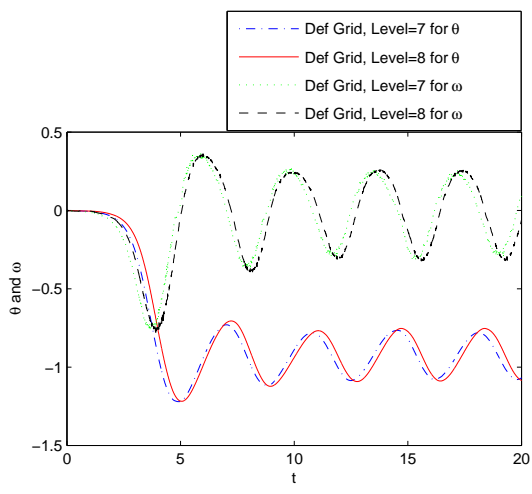

(a) Deformation meshes

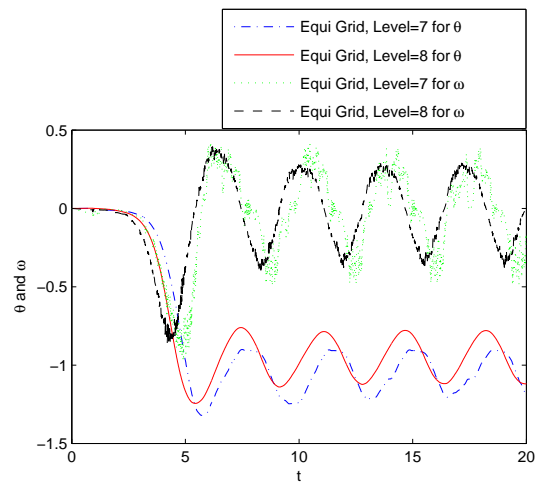

(b) Equidistance meshes

Fig. 2. The time history of the angle of incidence $\theta$ and the angular velocity $\omega$ of the induced rotating NACA0012 airfoil in a channel

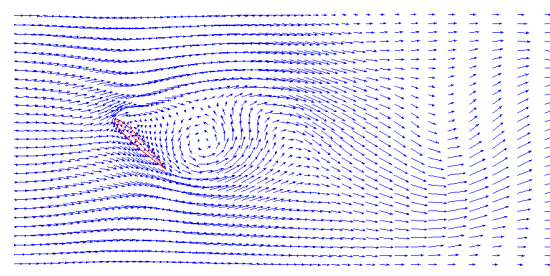

(a) Local vector field $(t=14.7)$

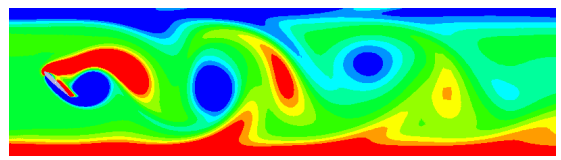

(c) Vorticity $(t=14.7)$

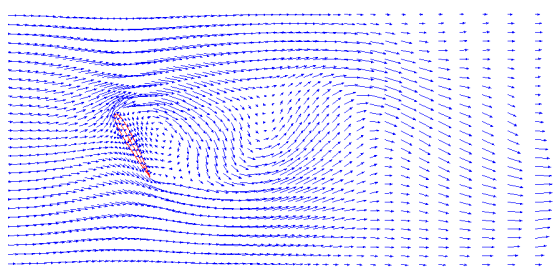

(b) Local vector field $(t=16.0)$

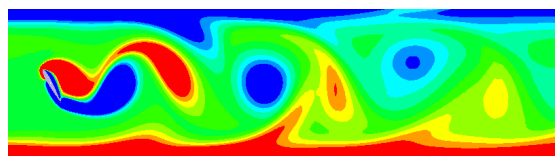

(d) Vorticity $(t=16.0)$

Fig. 3. Induced rotation of a NACA0012 airfoil in a channel

and $\mathrm{LEVEL}=8$, respectively. The vector field, vorticity distribution, norm of velocity, and pressure contour for the two instants $t=14.7$ and $t=16.0$ are given in Fig. 3 and Fig. 4. From these figures and pictures, we can see that the airfoil quickly reaches a periodic motion and intends to keep its broadside perpendicular to the in-flow direction which is a stable position for a noncircular rigid body settling in a channel at moderate Reynolds numbers. We observe that the results of the deformation meshes converge better to a mesh independent solution than those of the equidistance meshes, and are in excellent agreement with those obtained by Glowinski, Joseph and coauthors [3]. The results of the equidistance meshes exhibit too much numerical oscillation and 
lose stability since they cannot catch very well the velocity field close to the leading edge of the airfoil, which causes the numerical solution blew up near the leading edge of the airfoil. Obviously, good results and a significant accuracy improvement are achieved by using the grid deformation technique. It illustrates that the presented method can easily handle more complex shapes of rigid bodies and obtain more accurate and satisfying results than those without employing the grid deformation method.

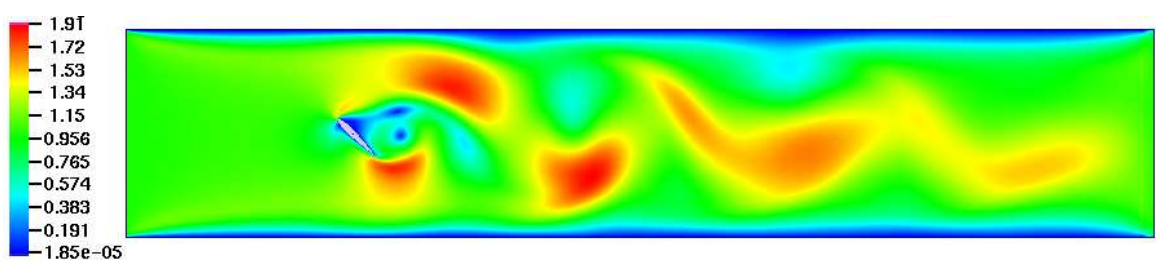

(a) Norm of velocity $(t=14.7)$

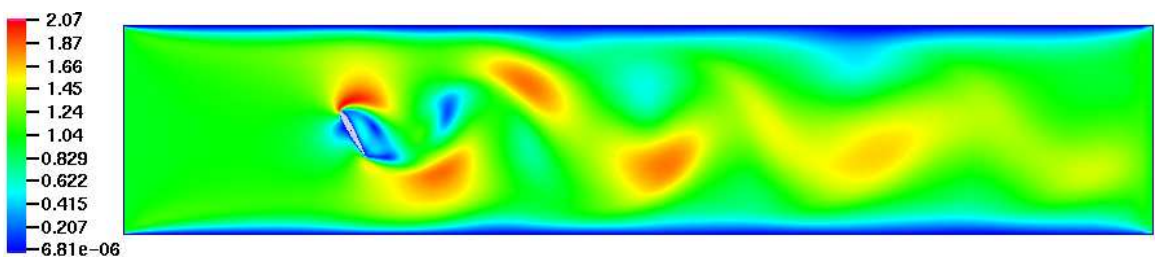

(b) Norm of velocity $(t=16.0)$

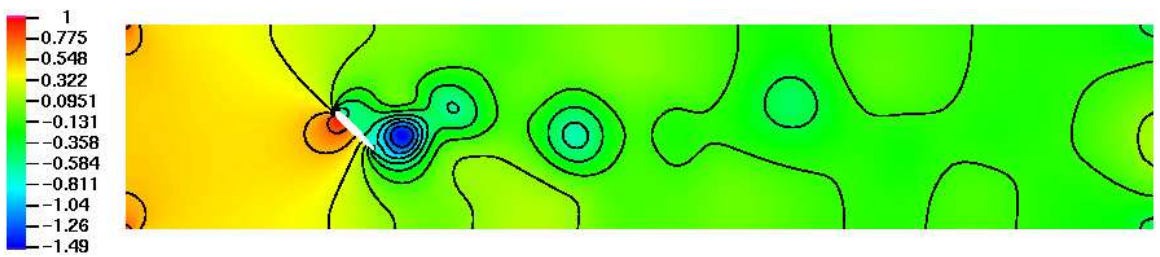

(c) Pressure $(t=14.7)$

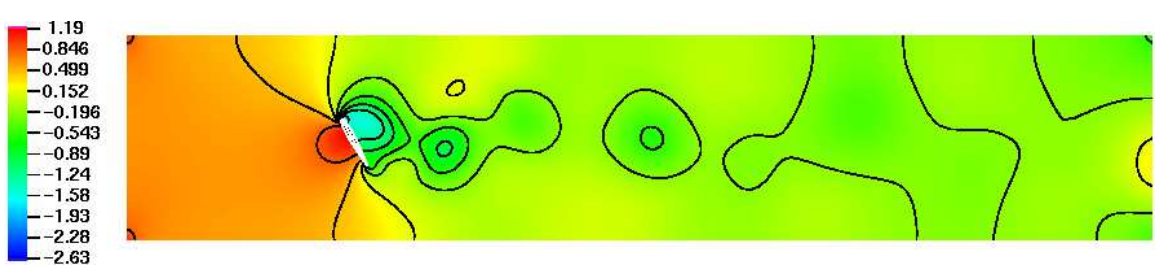

(d) Pressure $(t=16.0)$

Fig. 4. Induced rotation of a NACA0012 airfoil in a channel 


\section{Conclusions}

We have presented the combination of the multigrid fictitious boundary method (FBM) and the grid deformation method for the simulations of coupled fluid-rigid solid problems. Deformed grids, created from an arbitrary starting mesh, can preserve the topology of the mesh and the underlying data structure, while at the same time, the grid points can be concentrated and aligned near the surfaces of the solid bodies. Therefore, a better approximation of the solid surfaces is achieved. Incorporating the grid deformation method with the multigrid fictitious boundary method, an ALE formulation with a multigrid finite element solver is applied to solve the fluid flow, and the rigid body can move freely through the deformed meshes. Numerical examples have illustrated that the accuracy for dealing with the interaction between the fluid and the solid body by the presented method can be significantly improved.

\section{References}

1. Hu, H.H., Joseph, D.D., Crochet, M.J.: Direct Simulation of Fluid Particle Motions. Theor. Comp. Fluid Dyn., 3, 285-306 (1992)

2. Maury, B.: Direct Simulations of 2D Fluid-Particle Flows in Biperiodic Domains. J. Comput. Phy., 156, 325-351 (1999)

3. Glowinski, R., Pan, T.W., Hesla, T.I., Joseph, D.D., Periaux, J.: A Fictitious Domain Approach to the Direct Numerical Simulation of Incompressible Viscous Flow Past Moving Rigid Bodies: Application to Particulate Flow. J. Comput. Phy., 169, 363-426 (2001)

4. Turek, S., Wan, D.C., Rivkind, L.S.: The Fictitious Boundary Method for the Implicit Treatment of Dirichlet Boundary Conditions with Applications to Incompressible Flow Simulations. Challenges in Scientific Computing, Lecture Notes in Computational Science and Engineering, Vol. 35, Springer, 37-68 (2003)

5. Wan, D.C., Turek, S.: Direct Numerical Simulation of Particulate Flow via Multigrid FEM Techniques and the Fictitious Boundary Method. Int. J. Numer. Method in Fluids, in press (2005)

6. Cai, X.X., Fleitas, D., Jiang, B., Liao, G.: Adaptive Grid Generation Based on Least-Squares Finite-Element Method. Computers and Mathematics with Applications, 48(7-8), 1077-1086 (2004)

7. Grajewski, M., Köster, M., Kilian, S. and Turek, S.: Numerical Analysis and Practical Aspects of a Robust and Efficient Grid Deformation Method in the Finite Element Context. submitted to SISC, (2005)

8. Dacorogna, B., Moser, J.: On a Partial Differential Equation Involving the Jacobian Determinant. Annales de le Institut Henri Poincare, 7, 1-26 (1990)

9. Turek, S.: Efficient Solvers for Incompressible Flow Problems. Springer Verlag, Berlin-Heidelberg-New York, (1999)

10. Turek, S., et al.: FEATFLOW-Finite element software for the incompressible Navier-Stokes equations: User Manual, Release 1.2, University of Dortmund, (1999) 